C-A/AP/\#208

August 2005

\title{
Revision of the Adjusted Field Profile Estimate Criterion
}

Alessandro G. Ruggiero

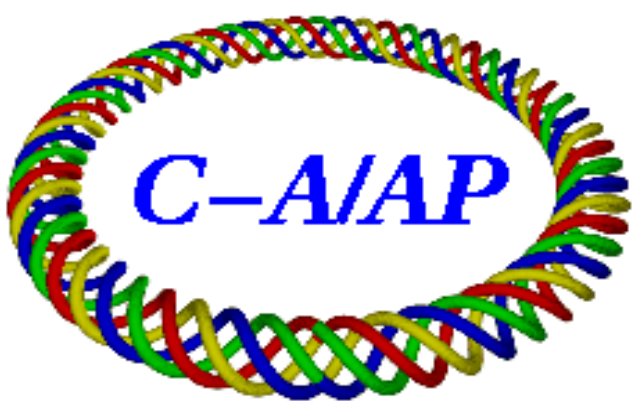

Collider-Accelerator Department

Brookhaven National Laboratory

Upton, NY 11973 


\title{
Revision of the Adjusted Field Profile Estimate Criterion
}

\author{
Alessandro G. Ruggiero \\ Brookhaven National Laboratory
}

July 25, 2005

\section{Introduction}

In a recent report [1] we have outlined a method for the cancellation of chromatic effects in a Fixed-Field Alternating-Gradient (FFAG) Accelerator. The method requires the estimate of the magnetic field profile, the so-called Adjusted Field Profile (AFP), that has to be established along the length and across the width of the ring magnets, in order to achieve essentially a constant betatron tune across the momentum aperture. The method was then applied to a particular FFAG project for the upgrade of the Alternating Gradient Synchrotron (AGS) [2] at Brookhaven National Laboratory (BNL), and thereafter we performed tracking to determine betatron acceptance. During tracking studies we noticed a considerable amount of beam loss and, counter to our expectations, with the original AFP applied, there were very large variations of tune across the momentum aperture also in the limit of small betatron oscillation amplitude. We reviewed therefore our original theoretical approach for the AFP determination and found a major flaw that had thus to be corrected. The new approach, hopefully now correct, for the determination of the AFP is outlined here. We also report the results of tracking using the new approach. It was found that indeed the tune variation across the momentum aperture is now minimal and acceptable in the limit of vanishing betatron oscillation amplitude. But we also found that, because of the large field nonlinearities involved, there was a dramatic betatron dynamic aperture reduction that makes our approach not quite desirable.

\section{Equation of Motion on the Horizontal Plane}

The linearized equation of motion of a proton moving in the FFAG accelerator on the horizontal plane is

$\mathrm{x}^{\prime \prime}+\mathrm{h}^{2}[1+\mathrm{n}(\mathrm{x}, \mathrm{s})] \mathrm{x} /(1+\delta)=\mathrm{h} \delta /(1+\delta)$

where $\mathrm{x}$ is the radial displacement from a reference trajectory corresponding to a reference momentum $\mathrm{p}_{0}$. A prime denotes derivative with respect to the longitudinal path length $\mathrm{s}$ along the reference trajectory that is also described by the curvature $h=h(s) . \delta=$ $\left(\mathrm{p}-\mathrm{p}_{0}\right) / \mathrm{p}_{0}$ is the relative momentum deviation from the reference value, and $\mathrm{n}(\mathrm{x}, \mathrm{s})$ is the field index taken to be generally a function of both path length $s$ and radial displacement $\mathrm{x}$. The relation between the field index $\mathrm{n}$ and the gradient $\mathrm{G}$ is

$$
\mathrm{h}^{2} \mathrm{n}(\mathrm{x}, \mathrm{s}) \quad=\quad \mathrm{G}(\mathrm{x}, \mathrm{s}) / \mathrm{B} \rho
$$

where $\mathrm{B} \rho$ is the magnetic rigidity of the reference particle with momentum $\mathrm{p}_{0}$. 
The general solution of Eq. (1)

$\mathrm{x}(\mathrm{s})=\mathrm{x}_{\mathrm{c}}(\mathrm{s})+\mathrm{u}(\mathrm{s})$

is given as the sum of the periodic closed orbit $\mathrm{x}_{\mathrm{c}}(\mathrm{s})$ and of the free betatron oscillation $u(s)$. Both are also function of the momentum deviation $\delta$. Insertion of Eq. (3) in (1) gives

$\mathrm{x}_{\mathrm{c}}{ }^{\prime \prime}+\mathrm{u}^{\prime \prime}+\mathrm{h}^{2}\left[1+\mathrm{n}\left(\mathrm{x}_{\mathrm{c}}+\mathrm{u}, \mathrm{s}\right)\right]\left(\mathrm{x}_{\mathrm{c}}+\mathrm{u}\right) /(1+\delta)=\mathrm{h} \delta /(1+\delta)$

By definition the closed orbit solution satisfies the equation

$\mathrm{x}_{\mathrm{c}}^{\prime \prime}+\mathrm{h}^{2}\left[1+\mathrm{n}\left(\mathrm{x}_{\mathrm{c}}, \mathrm{s}\right)\right] \mathrm{x}_{\mathrm{c}} /(1+\delta)=\mathrm{h} \delta /(1+\delta)$

After linearization the free betatron oscillation satisfies the equation

$\mathrm{u}^{\prime \prime}+\mathrm{h}^{2}\left[1+\mathrm{n}\left(\mathrm{x}_{\mathrm{c}}, \mathrm{s}\right)+\mathrm{x}_{\mathrm{c}} \mathrm{dn}\left(\mathrm{x}_{\mathrm{c}}, \mathrm{s}\right) / \mathrm{dx}_{\mathrm{c}}\right] \mathrm{u} /(1+\delta)=0$

It is convenient to write

$\mathrm{n}\left(\mathrm{x}_{\mathrm{c}}, \mathrm{s}\right) \quad=\quad \mathrm{n}_{0}\left[1+\mathrm{f}\left(\mathrm{x}_{\mathrm{c}}, \mathrm{s}\right)\right]$

where

$\mathrm{h}^{2} \mathrm{n}_{0} \quad=\quad \mathrm{G}_{0} / \mathrm{B} \rho$

is the field index on the reference trajectory at $\mathrm{x}_{\mathrm{c}}=0$ for any longitudinal location $\mathrm{s}$. Then Eq. (6) becomes

$\mathrm{u}^{\prime \prime}+\mathrm{h}^{2} \mathrm{u} /(1+\delta)+\mathrm{h}^{2} \mathrm{n}_{0}\left[1+\mathrm{f}\left(\mathrm{x}_{\mathrm{c}}, \mathrm{s}\right)+\mathrm{x}_{\mathrm{c}} \mathrm{df}\left(\mathrm{x}_{\mathrm{c}}, \mathrm{s}\right) / \mathrm{dx}_{\mathrm{c}}\right] \mathrm{u} /(1+\delta)=0$

\section{Chromaticity Cancellation}

In order to flatten the dependence of the betatron tune with momentum we require

$f\left(x_{c}, s\right)+x_{c} d f\left(x_{c}, s\right) / d_{c}=\delta$

so that Eq. (9) becomes

$\mathrm{u}^{\prime \prime}+\mathrm{h}^{2} \mathrm{u} /(1+\delta)+\mathrm{h}^{2} \mathrm{n}_{0} \mathrm{u}=0$

and, at the same time, the closed orbit Eq. (5) changes to

$\mathrm{x}_{\mathrm{c}}{ }^{\prime \prime}+\mathrm{h}^{2}\left(1+\mathrm{n}_{0}\right) \mathrm{x}_{\mathrm{c}} /(1+\delta)+\mathrm{h}^{2} \mathrm{n}_{0} \mathrm{f}\left(\mathrm{x}_{\mathrm{c}}, \mathrm{s}\right) \mathrm{x}_{\mathrm{c}} /(1+\delta)=\mathrm{h} \delta /(1+\delta)$ 
In principle Eq. (12) can be solved for the closed orbit $\mathrm{x}_{\mathrm{c}}=\mathrm{x}_{\mathrm{c}}(\mathrm{s}, \delta)$ as a function of $\mathrm{s}$ and $\delta$. At any location $\mathrm{s}$ there is a one-to-one correspondence between $\mathrm{x}_{\mathrm{c}}$ and $\delta$, so that by inversion one formally derives $\delta=\delta\left(\mathrm{x}_{\mathrm{c}}, \mathrm{s}\right)$ that can replace the right hand side of Eq. (10). The equation so obtained can then be solved to derive $f\left(x_{c}, s\right)$. We shall make the assumption that $\mathrm{x}_{\mathrm{c}}(\mathrm{s}, 0)=\delta(0, \mathrm{~s})=0$ so that the formal integration of Eq. (10) gives

$\mathrm{f}\left(\mathrm{x}_{\mathrm{c}}, \mathrm{s}\right)=\int_{0}^{\mathrm{x}_{\mathrm{c}}} \delta(\xi, \mathrm{s}) \mathrm{d} \tilde{\xi} / \mathrm{x}_{\mathrm{c}}$

The two Eq.s (12 and 13) combined are of no immediate integration. We need to solve them in steps. In linear approximation $\mathrm{x}_{\mathrm{c}}=\eta(\mathrm{s}) \delta$ so that

$\mathrm{f}\left(\mathrm{x}_{\mathrm{c}}, \mathrm{s}\right) \approx \int \xi \mathrm{d} \tilde{\xi} / \eta(\mathrm{s}) \mathrm{x}_{\mathrm{c}}=\mathrm{x}_{\mathrm{c}} / 2 \eta(\mathrm{s})=\delta / 2$

where the integration limits are 0 and $\mathrm{x}_{\mathrm{c}}$. Inserting this in Eq. (12) gives

$\mathrm{x}_{\mathrm{c}}^{\prime \prime}+\mathrm{h}^{2} \mathrm{x}_{\mathrm{c}} /(1+\delta)+\mathrm{h}^{2} \mathrm{n}_{0}(1+\alpha \delta) \mathrm{x}_{\mathrm{c}} /(1+\delta)=\mathrm{h} \delta /(1+\delta)$

where for the moment $\alpha \approx 1 / 2$.

Eq.(15) is similar to the one we have solved earlier [1] for the estimate of the AFP, except that then we have used $\alpha=1$. That led to an exceedingly large tune variation and even beam instability because Eq. (10) cannot be satisfied identically with $\alpha=1$. Our approach is to solve first Eq. (15) with $\alpha=1 / 2$ and thus derive an approximate value of $x_{c}$ $=\mathrm{x}_{\mathrm{c}}(\mathrm{s}, \delta)$ that can be inverted to derive again $\delta=\delta\left(\mathrm{x}_{\mathrm{c}}, \mathrm{s}\right)$. Insertion of the new estimate in Eq. (13) leads to a new value of $f\left(x_{c}, s\right)$ and the procedure is then repeated iteratively.

A more accurate estimate is obtained by using the quadratic approximation $\mathrm{x}_{\mathrm{c}}=\eta(\mathrm{s}) \delta+$ $\psi(\mathrm{s}) \delta^{2}$ that inserted in Eq. (10) gives

$\mathrm{f}\left(\mathrm{x}_{\mathrm{c}}, \mathrm{s}\right) \approx \int\left[-\eta+\left(\eta^{2}+4 \psi \xi\right)^{1 / 2}\right] \mathrm{d} \xi / 2 \psi(\mathrm{s}) \mathrm{x}_{\mathrm{c}}$

where the integration limits are again 0 and $x_{c}$. Performing the integration and reinserting the expression of $\mathrm{x}_{\mathrm{c}}$ as a function of $\delta$ yields

$\mathrm{f}\left(\mathrm{x}_{\mathrm{c}}, \mathrm{s}\right) \approx(\delta / 2)(1+4 \psi \delta / 3 \eta) /(1+\psi \delta / \eta) \approx(\delta / 2)(1+\psi \delta / 3 \eta)$.

In the limit $\psi \delta / \eta<<1$ we recover $\mathrm{f}\left(\mathrm{x}_{\mathrm{c}}, \mathrm{s}\right) \approx(\delta / 2)$. Otherwise, there is a minor dependence with $\delta$ accompanied by a variation with the longitudinal position s. 


\section{General Equations of Motion}

Eq. (1) describes the motion along the horizontal direction on the $y=0$ mid-plane. We assume here that there is no vertical bending, and that the reference trajectory lies entirely on the $y=0$ mid-plane. To study the more general motion both along the radial displacement $\mathrm{x}$ and the displacement $\mathrm{y}$ from the mid-plane, it is convenient to introduce complex coordinates. Let $\mathrm{z}=\mathrm{x}+\mathrm{i} \mathrm{y}$ be the particle position, and $\mathrm{B}=\mathrm{B}_{\mathrm{y}}+\mathrm{i} \mathrm{B}_{\mathrm{x}}$ the complex magnetic field of components $\mathrm{B}_{\mathrm{x}}$ along the radial direction and the vertical component $\mathrm{B}_{\mathrm{y}}$. We can also relate the complex field index $\mathrm{n}(\mathrm{z}, \mathrm{s})$ to the bending field

$$
\mathrm{B}(\mathrm{z}, \mathrm{s})=\mathrm{B}_{0}[1+\mathrm{hn}(\mathrm{z}, \mathrm{s}) \mathrm{z}]
$$

where $\mathrm{B}_{0}$ is the bending field on the reference trajectory $(\mathrm{x}=\mathrm{y}=0)$. If higher-order terms are neglected, the general equations of motion are

$$
\begin{array}{ll}
\mathrm{x}^{\prime \prime}+\mathrm{h}^{2}\{\mathrm{x}+\operatorname{Re}[\mathrm{n}(\mathrm{z}, \mathrm{s}) \mathrm{z}]\} /(1+\delta) & =\mathrm{h} \delta /(1+\delta) \\
\mathrm{y}^{\prime \prime}-\mathrm{h}^{2} \operatorname{Im}[\mathrm{n}(\mathrm{z}, \mathrm{s}) \mathrm{z}] /(1+\delta) & =0
\end{array}
$$

The reference trajectory is defined by $\mathrm{x}=\mathrm{y}=\delta=0$. The general solution of Eq.s ( 19 and 20) can be written as $\mathrm{x}=\mathrm{x}_{\mathrm{c}}+\mathrm{u}$ and $\mathrm{y}=\mathrm{y}_{\mathrm{c}}+\mathrm{v}$, where $\mathrm{u}$ and $\mathrm{v}$ represent the free betatron oscillations around the closed orbit of coordinates $x_{c}$ and $y_{c}$. Because of the mid-plane symmetry $y_{c}=0$ identically, and $x_{c}$ is given by Eq. (5). The free radial betatron oscillation on the mid-plane $y=0$, satisfies the equation

$$
\mathrm{u}^{\prime \prime}+\mathrm{h}^{2}\left\{\mathrm{u}+\operatorname{Re}\left[\mathrm{n}\left(\mathrm{x}_{\mathrm{c}}+\mathrm{u}, \mathrm{s}\right)\left(\mathrm{x}_{\mathrm{c}}+\mathrm{u}\right)\right]-\operatorname{Re}\left[\mathrm{n}\left(\mathrm{x}_{\mathrm{c}}, \mathrm{s}\right) \mathrm{x}_{\mathrm{c}}\right]\right\} /(1+\delta)=0 .
$$

Linearization of this equation gives

$$
\mathrm{u}^{\prime \prime}+\mathrm{h}^{2}\left[1+\mathrm{n}_{R e}\left(\mathrm{x}_{\mathrm{c}}, \mathrm{s}\right)+\mathrm{x}_{\mathrm{c}} \mathrm{dn}_{R e}\left(\mathrm{x}_{\mathrm{c}}, \mathrm{s}\right) / \mathrm{d} \mathrm{x}_{\mathrm{c}}\right] \mathrm{u} /(1+\delta)=0 .
$$

This equation is similar to Eq. (6) after having noticed that $\mathrm{n}_{R e}\left(\mathrm{x}_{\mathfrak{c}}, \mathrm{s}\right)=\mathrm{n}\left(\mathrm{x}_{\mathfrak{c}}, \mathrm{s}\right)$ and $\mathrm{n}_{I m}$ $\left(\mathrm{x}_{\mathrm{c}}, \mathrm{s}\right)=0$. We have already discussed this equation, and determined that, in order to flatten the momentum dependence of the radial betatron oscillation for amplitudes, the condition represented by Eq. (10) is to be fulfilled, after having associated the function $\mathrm{f}\left(\mathrm{x}_{\mathrm{c}}, \mathrm{s}\right)$ to the field index $\mathrm{n}\left(\mathrm{x}_{\mathrm{c}}, \mathrm{s}\right)$ according to Eq. (7).

We follow a similar approach also for Eq. (20) that we linearly expand around $y=0$ in the limit of vanishing radial betatron oscillation amplitude $(u=0)$. We obtain

$$
\mathrm{v}^{\prime \prime}-\mathrm{h}^{2}\left[\mathrm{n}\left(\mathrm{x}_{\mathrm{c}}, \mathrm{s}\right)+\mathrm{x}_{\mathrm{c}} \mathrm{dn}\left(\mathrm{x}_{\mathrm{c}}, \mathrm{s}\right) / \mathrm{d} \mathrm{x}_{\mathrm{c}}\right] \mathrm{v} /(1+\delta)=0
$$

Thus the same condition of Eq. (10) is to be fulfilled to flatten the momentum dependence of the vertical betatron oscillations in the limit of small amplitudes. The main difference between Eq.s (22 and 23) is the curvature term that still carries momentum dependence for the horizontal oscillations and not for the vertical ones. 


\section{FFAG for the AGS Upgrade}

For convenience we report here the main parameters of the FFAG accelerator proposed as the new injector of the AGS at BNL [2]. We shall use again the parameter $\delta$ to define the momentum of any particle $\mathrm{p}=\mathrm{p}_{0}(1+\delta)$. At injection $\delta=0$ and that is taken also to be the reference trajectory. The beam kinematics parameters are given in Table 1. The lattice is made of a sequence of identical periods shown in Figure 1. Table 2 provides a summary of the magnet parameters at injection. Table 3 lists the main lattice parameters for the injection orbit. The main lattice functions are displayed in Figure 2 also for the injection orbit.

Table 1. Proton Beam Kinematic Parameters

\begin{tabular}{lll} 
& Injection & Extraction \\
Kinetic Energy, MeV & \multicolumn{1}{c}{400} & \multicolumn{1}{c}{1,500} \\
$\beta$ & 0.713056 & 0.922996 \\
$\gamma$ & 1.42632 & 2.59868 \\
Momentum, MeV/c & 954.263 & $2,250.51$ \\
Magnetic Rigidity, kG-m & 31.8308 & 75.0691 \\
Momentum Deviation, $\delta$ & 0 & 1.36
\end{tabular}

Table 2. Magnet Parameters for the Injection Orbit

\begin{tabular}{lll} 
Magnet Type & \multicolumn{1}{c}{ F } & \multicolumn{1}{c}{ D } \\
Arc Length, m & 0.70 & 0.70 \\
Bending Field B, kG & -0.78409 & 1.8345 \\
Gradient G, kG/m & 26.5817 & -23.2956 \\
Bending Radius $\rho, \mathrm{m}$ & -40.5958 & 17.3512 \\
Bending Angle, mrad & -17.2432 & 80.6862
\end{tabular}

Table 3. The AGS-FFAG Parameters for the Reference Trajectory

Circumference

Number of Periods

Period Length

Period Structure

Short Drift, g

Long Drift, S (total)

$\beta_{\mathrm{H}} \max ($ in $\mathrm{S})$

$\beta_{\mathrm{V}} \max ($ in D)

$\eta \max ($ in $\mathrm{S}$ )

Phase Adv. / Period, H/V

Betatron Tunes, $\mathrm{H} / \mathrm{V}$

Natural Chromaticity, H/V

Transition Energy, $\gamma_{\mathrm{T}}$
$807.091 \mathrm{~m}$

136

$5.93449 \mathrm{~m}$

S F $g$ D $g$ g F

$0.30 \mathrm{~m}$

$2.53450 \mathrm{~m}$

$4.5733 \mathrm{~m}$

$11.7902 \mathrm{~m}$

$0.060 \mathrm{~m}$

$105.23^{\circ} / 99.935^{\circ}$

$39.755 / 37.755$

$-0.926 /-1.805$

$105.482 \mathrm{i}$ 


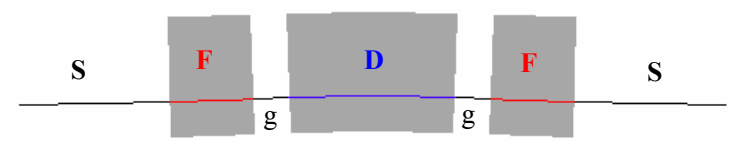

Figure 1. The AGS-FFAG Period (FDF) and the Injection Trajectory.

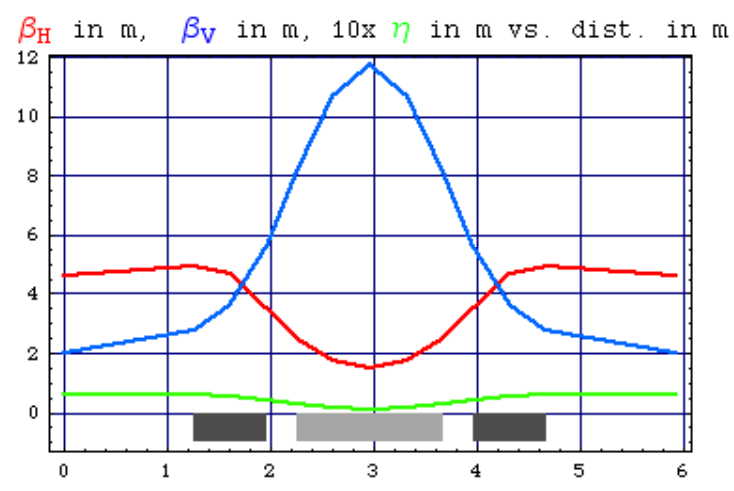

Figure 2. The AGS-FFAG Lattice Functions for the Injection Orbit $(\delta=0)$

\section{Linear Field Profile}

We first try a Linear Field Profile (LFP) across the width of the magnets that make the triplet period. The field distribution is shown in Figure 3. The field strength is modest and ranges between $-0.784 \mathrm{kG}$ and $3.794 \mathrm{kG}$ in the F-sector magnet, and between $1.835 \mathrm{kG}$ and $-1.400 \mathrm{kG}$ in the F-sector magnet. Magnets are typically quadrupoles with a radial offset to adjust the bending fields as required on the injection orbit. The location of closed orbits at different particle energies between injection and extraction are displayed along the length of half of a period in Figure 4. There is indeed a mirror symmetry point in the middle of the long straight $\mathrm{S}$ and another in the middle of the D-sector magnet. The magnet width required to accommodate at the least the entire momentum range during acceleration is thus $17 \mathrm{~cm}$ in the F-sector magnet and $14 \mathrm{~cm}$ in the D-sector magnet. We are adopting a Non-Scaling Lattice [3] and thus, with such a momentum variation and the LFP, a large betatron tune variation is also expected as it is shown in Figure 5. The corresponding variation of the betatron amplitude $\beta_{\mathrm{H}, \mathrm{V}}$ variation is displayed in Figure 6.

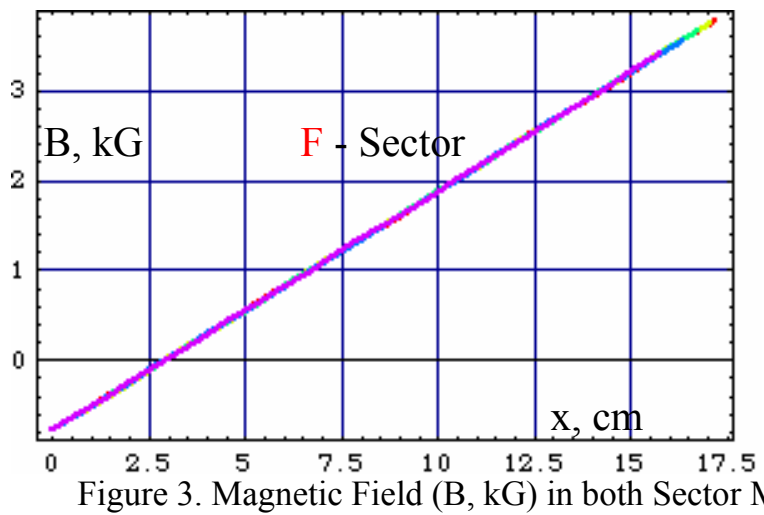

Figure 3. Magnetic Field (B, kG) in both Sector Magnets versus Radial Position (x, cm) with LFP.

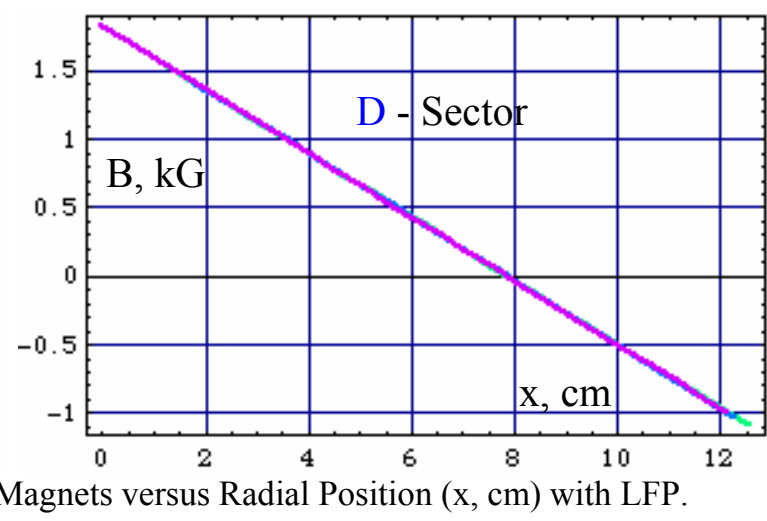




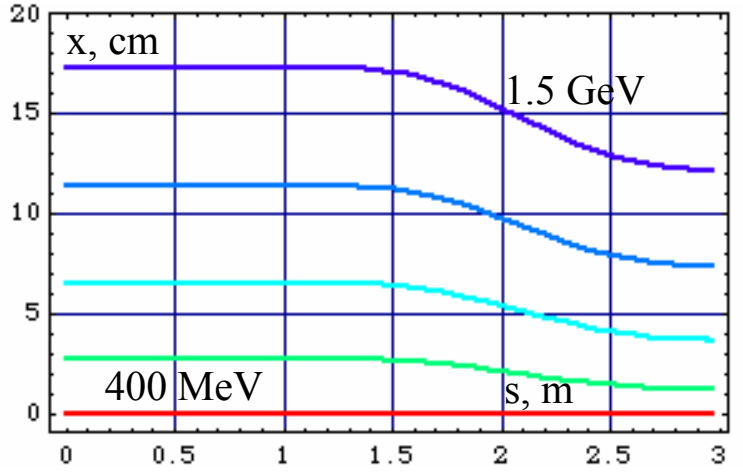

Figure 4. Closed Orbits $(\mathrm{x}, \mathrm{cm})$ at different Beam Energy along the length $(\mathrm{s}, \mathrm{m})$ of Half Period with LFP

Unfortunately, because of the large variation of the betatron tunes during acceleration, major structural resonances are encountered, despite of the large periodicity of the FFAG ring as it can be seen in the $\left(v_{\mathrm{H}}, v_{\mathrm{V}}\right)$ - plot of Figure 7. Structural resonances of $2 \mathrm{nd}$, 3rd and 4th order are shown. Those intersecting with the tune operation curve (in red) are of 3rd and 4th order. There are no crossings with 1 st and 2nd order strauctural resonances. Fortunately, because of the complete absence of non-linear magnetic fields, in the absence of imperfections and errors, no variation of the betatron tunes is expected with the amplitude of the betatron oscillations. Furthermore the tune diagram is covered with a denser population of resonaces that can be driven by errors and imperfections. These resonances are not shown in Figure 7. To determine the beam survival when crossing all types of resonances during acceleration it is necessary to resort to numerical simulation.

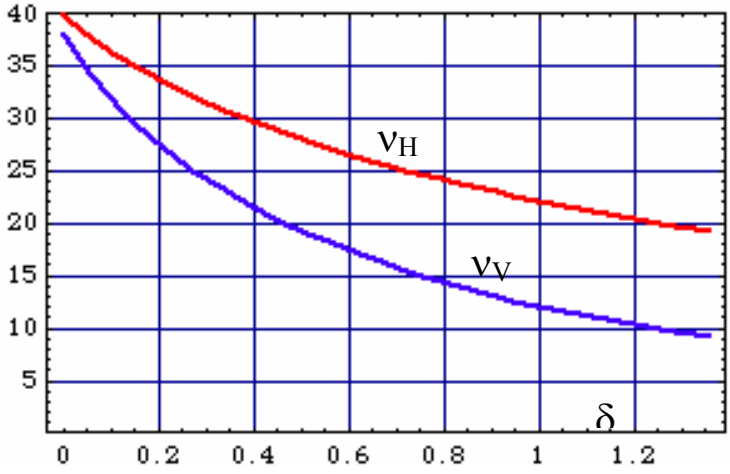

Figure 5. Betatron Tunes vs. Momentum $(\delta)$.

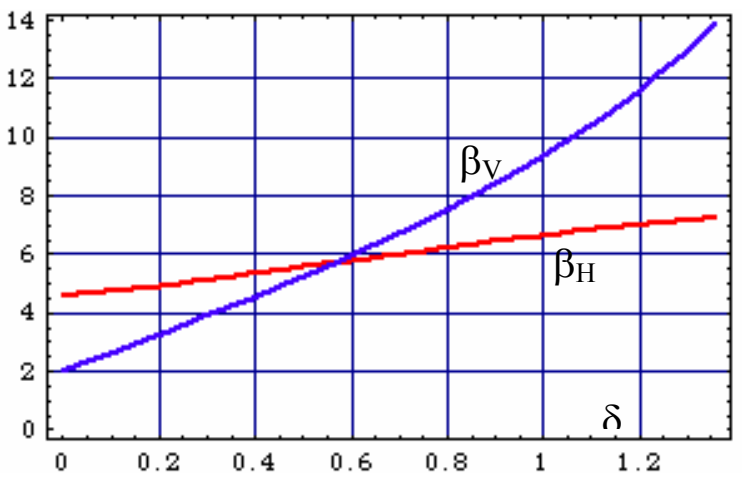

Figure 6. Betatron Amplitudes vs. Momentum ( $\delta)$

\section{Application of the Adjusted Field Profile}

We examine now the case when the Adjusted Field Profile (AFP) is applied, and check the consequences on the variation of the betatron tunes during acceleration. Let us first outline the procedure we have followed. We divided each magnet in a number of segments, 5 for the F-sector magnet and 10 for the D-sector magnet. In each segment we assumed a dependence of field index with the momentum deviation $\delta$ as follows

$\mathrm{n}(\delta)=\mathrm{n}_{0}\left(1+\mathrm{a} \delta+\mathrm{b} \delta^{2}\right)$

The coefficients a and $b$ vary from segment to segment and have been determined with the iteration method explained above. Their values are given in Table 4. Then Eq. (5) is solved with $\mathrm{n}\left(\mathrm{x}_{\mathrm{c}}, \mathrm{s}\right)$ replaced by $\mathrm{n}(\delta)$ given by Eq. $(24)$. The solution $\mathrm{x}_{\mathrm{c}}(\delta, \mathrm{s})$ is plotted in 


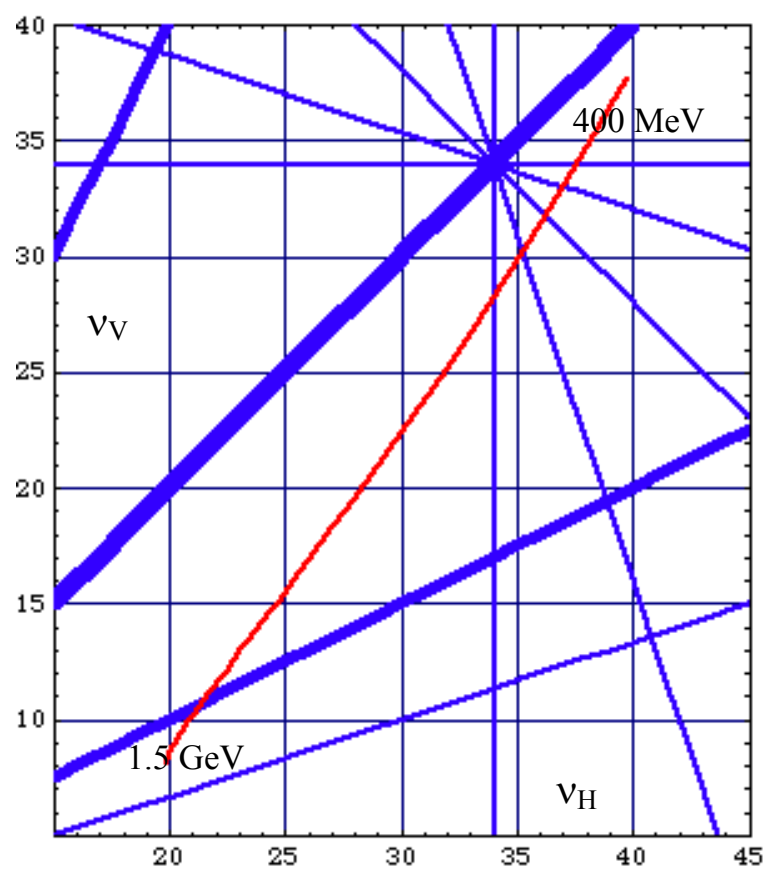

Figure 7. Tune Diagram for the LFP case with $2^{\text {nd }}, 3^{\text {rd }}$ and $4^{\text {th }}$ order systematic resonances

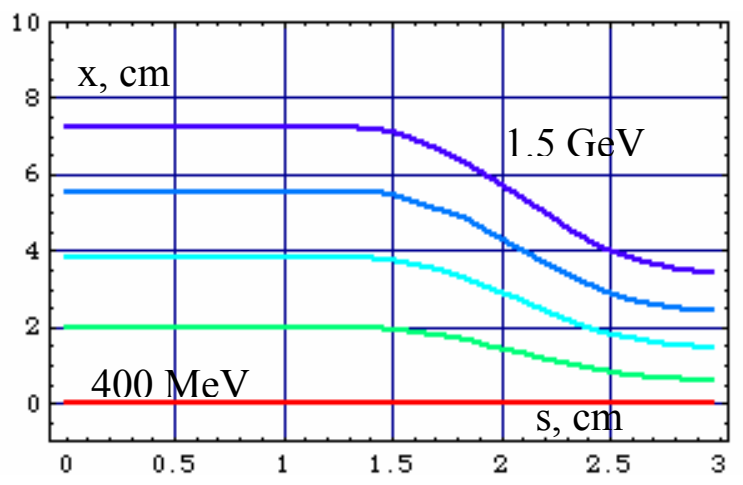

Figure 8. Closed Orbits $(\mathrm{x}, \mathrm{cm})$ at different Beam Energy along the length $(\mathrm{s}, \mathrm{m})$ of Half Period with AFP

Figure 8 for different values of beam energy between $400 \mathrm{MeV}$ and $1.5 \mathrm{GeV}$ versus the length of half a period. It is seen that now the magnet width required to accomodate the entire momentum acceleration is reduced to $7.2 \mathrm{~cm}$ in the Fsector magnet and $4.7 \mathrm{~cm}$ in the D-sector magnet; that is almost a factor of two when compared to the case of a LFP. Using MATHEMATICA [4] the solution $\mathrm{x}_{\mathrm{c}}(\delta, \mathrm{s})$ is then inverted to derive $\delta=\delta\left(\mathrm{x}_{\mathrm{c}}, \mathrm{s}\right)$, and the required Adjusted Field Profile (AFP) is calculated by making use of Eq. (18) with

$$
\mathrm{n}(\mathrm{z}, \mathrm{s})=\mathrm{n}_{0}\left[1+\mathrm{a} \delta(\mathrm{z}, \mathrm{s})+\mathrm{b} \delta(\mathrm{z}, \mathrm{s})^{2}\right]
$$

Table 4. Coefficients of $\delta$ dependence of Field-Index

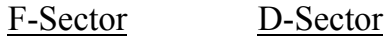

$\begin{array}{lrccc}\text { Segment } & \mathrm{a} & \mathrm{b} & \mathrm{a} & \mathrm{b} \\ & & & & \\ 1 & 0.500 & -0.0140 & 0.497 & 0.0171 \\ 2 & 0.500 & -0.0132 & 0.493 & 0.0271 \\ 3 & 0.500 & -0.0116 & 0.488 & 0.0376 \\ 4 & 0.500 & -0.0090 & 0.482 & 0.0471 \\ 5 & 0.499 & -0.0052 & 0.478 & 0.0531\end{array}$

The AFP so determined along the width of each magnet is plotted in Figure 9 at five different longitudinal locations in each magnet. The field ranges between $-0.78 \mathrm{kG}$ and $1.83 \mathrm{kG}$ in the F-sector magnet, and between $2.42 \mathrm{kG}$ and $-0.07 \mathrm{kG}$ in the $\mathrm{D}$-sector magnet. A large nonlinearity of the field is clearly noticeable. In the middle of each magnet segment the field expressions given by MATHEMATICA are fitted with a multiple expansion up to and including $z^{5}$ terms. The next step, always using the same code, is to integrate Eq.s (22 and 23) by tracking. This is done by lumping the nonlinearities in the middle of each magnet segment. Tracking allows then estimating of the betatron tunes versus momentum deviation, and for different betatron oscillation amplitudes both in the radial and vertical direction. The betatron tune and amplitude variation across the momentum range are plotted in Figures 10 and 11 . The tune diagram of Figure 12 shows that the tune range during acceleration is well confined, and that nearby there are no major structural resonances, the lowest order of them crossing the tune range is 14. All the other resonances shown in Figure 12 are of lower order but do not cross the tune range during acceleration. There are of course other 

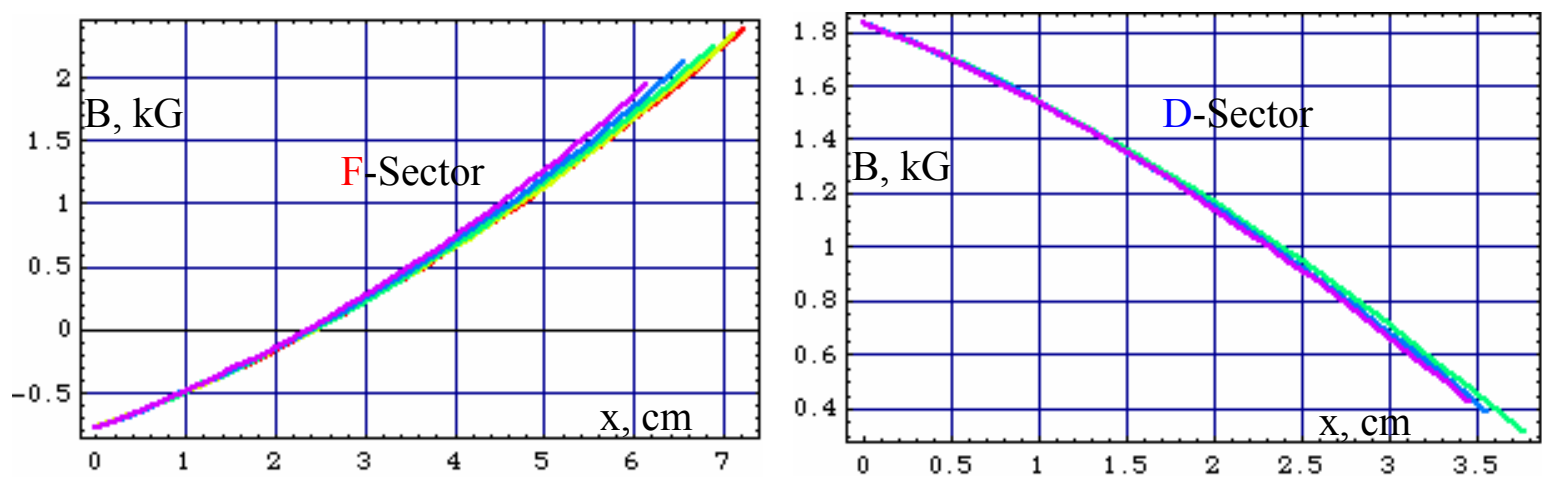

Figure 9. Magnetic Field (B, kG) in both Sector Magnets versus Radial Position (x, cm) with AFP.

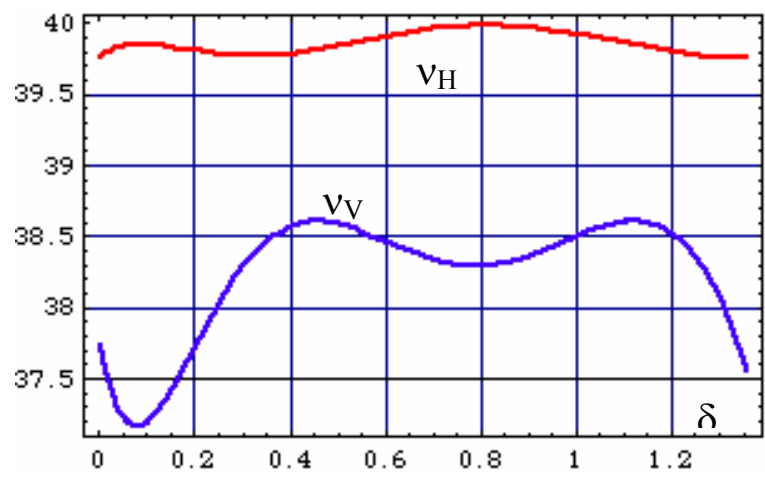

Figure 10. Betatron Tunes vs. Momentum $(\delta)$ with AFP

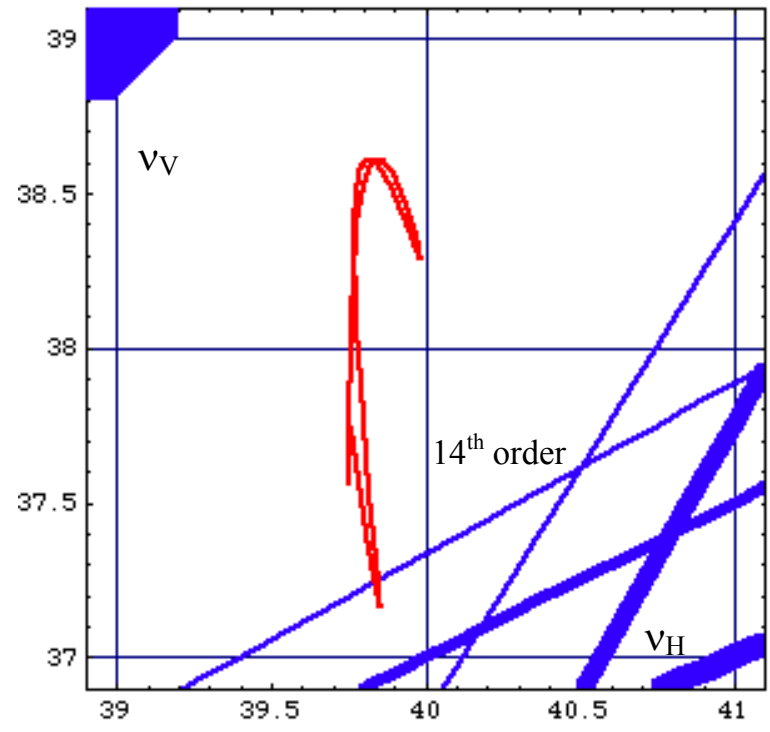

Figure 12. Tune Diagram with AFP

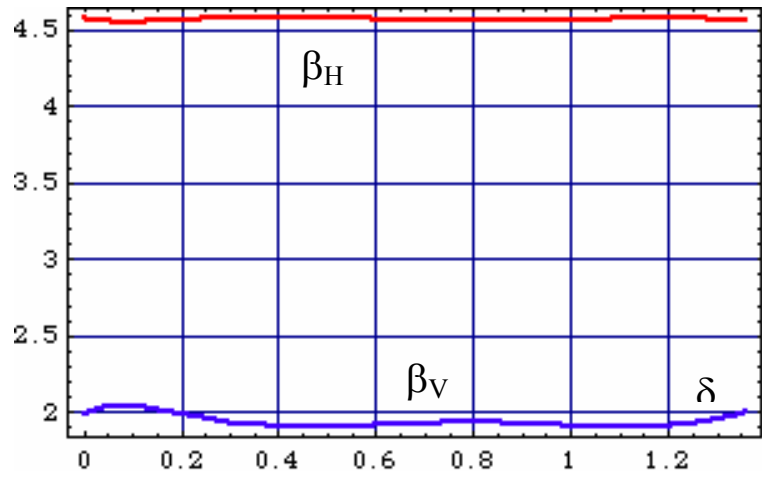

Figure 11. Betatron Amplitudes vs. Momentum ( $\delta$ ) with AFP

resonances that can be driven by magnet misalignment and imperfections and that cover more densily the tune diagram of Figure 12. These resonances are not shown in the Figure and need to be treated independently with an appropriate error analysis. It is also to be taken into account in the estimating of the beam survival that all types of resonances are crossed rather fast during acceleration in the FFAG ring.

The previous results were obtained including a variation of the AFP along the length of each magnet. To simplify the construction of the magnets we have also assumed an AFP distribution constant along the length of the magnets by replacing the actual values with their averages. The compactness of the closed orbits versus momentum does not change and it is still given by Figure 8 . There is though an appretiable change, especially in the vertical plane, in the 
variation of the betatron tunes and amplitudes as shown in Figures 13 and 14. But the change is still acceptable as only fewer structural resonances are crossed and all are of very high order. The new tune digram is shown in Figure 15. A part from the harmless $v_{\mathrm{H}}$ $=v_{\mathrm{V}}$ resonance the only other one crossing the tune operating curve (in red) is of order 18.

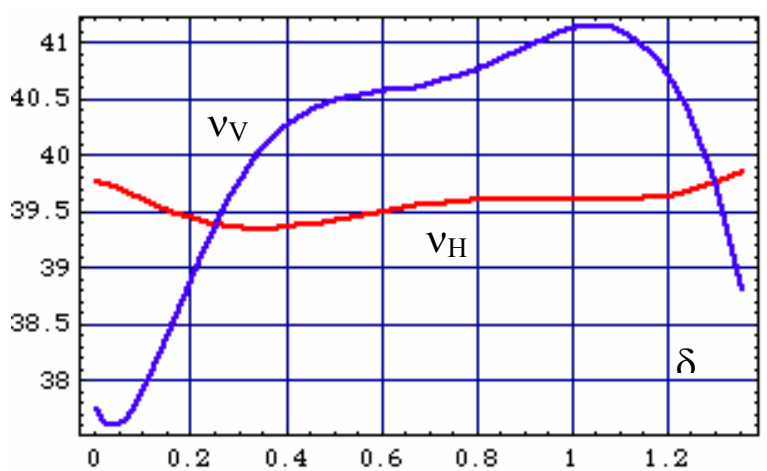

Figure 13. Betatron Tunes vs. Momentum $(\delta)$ with constant AFP

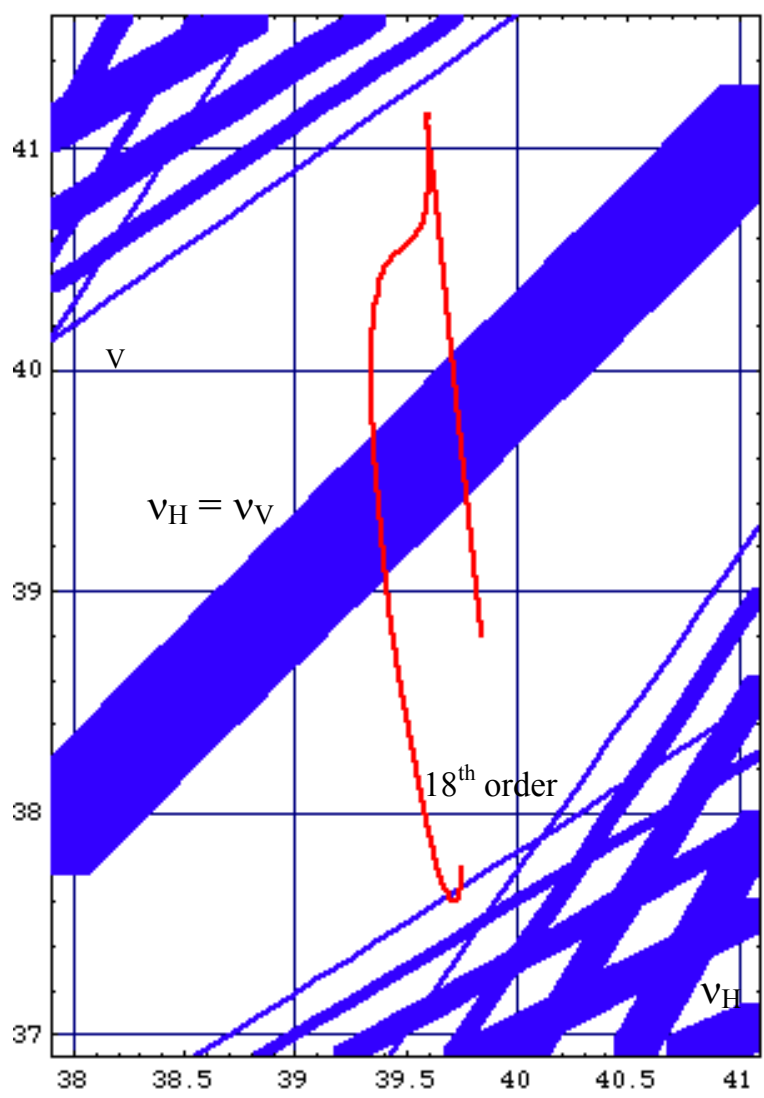

Figure 15. Tune Diagram with constant AFP

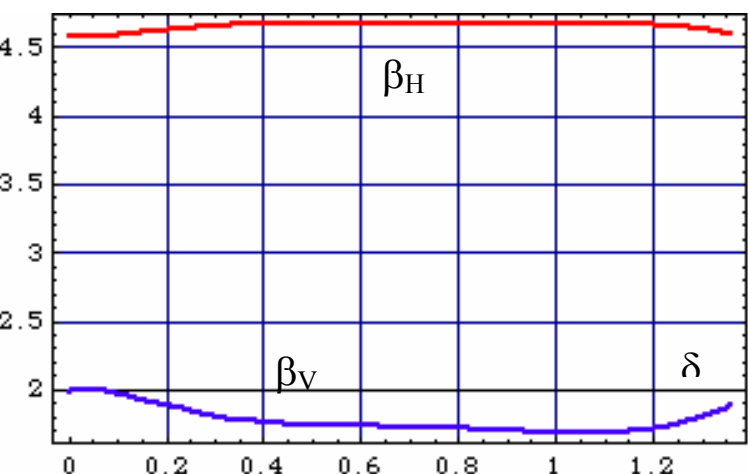

Figure 14. Betatron Amplitudes vs. Momentum ( $\delta$ ) with constant AFP

\section{Dependence of Tune with Amplitude}

The AFP introduce a significant amount of field nonlinearity in the magnets as one can see by inspecting Figure 9. There is thus the concern that this will cause a large variation of betatron tunes with amplitude of motion, as indeed it has been demonstrated again with numerical tracking. The result are shown in Figures $16 \mathrm{a}$ and $\mathrm{b}$. The variation is excessive and there is a strong limitation of dynamical aperture to just few centimeters of oscillation amplitude. In the horizontal direction of motion the limit is encounter when the change of tune with amplitude, that is always positive, has reached the half-integral stopband $2 v_{\mathrm{H}}=136$. In the vertical plane the change can be either positive or negative and the limit is reached when either approaching the stopband $2 v_{\mathrm{V}}=136$ or the tune is fully depressed. This result makes the validity of the application of the AFP very questionable, unless methods are found by
lements the tune variation is reduced. 

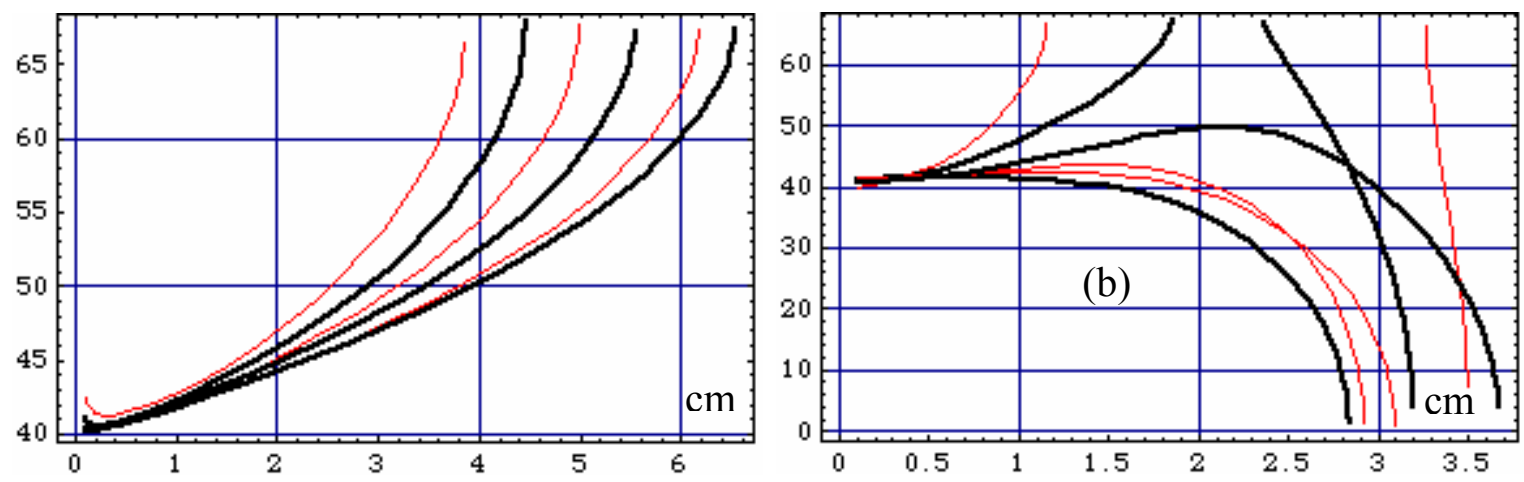

Figure 16. Variation of Horizontal (a) and Vertical (b) Betatron Tune with Oscillation Amplitude

\section{References}

[1] A. G. Ruggiero, "Adjusted Field Profile for the Chromaticity Cancellation in a FFAG Accelerator”, FFAG’04 Workshop Proceedings, October 13-16, 2004, KEK, Tsukuba, Japan.

[2] A. G. Ruggiero, "Feasibility Study of a 1.5-GeV Proton FFAG in the AGS Tunnel", BNL internal report C-A/AP/\#157, June 2004.

[3] A.G. Ruggiero, Design Criteria of a Proton FFAG Accelerator", FFAG'04 Workshop Proceedings, October 13-16, 2004, KEK, Tsukuba, Japan.

[4] MATHEMATICA, Wolfram Research, http://www.wolfram.com 